\title{
FORCE BALANCE IN CURRENT SHEETS IN COLLISIONLESS PLASMA
}

\section{O.V. Mingalev}

Polar Geophysical Institute RAS, Apatity, Russia,mingalev_o@pgia.ru Murmansk Arctic State University, Apatity,Russia,mingalev_o@pgia.ru

\section{P.V. Setsko}

Polar Geophysical Institute RAS, Apatity, Russia, setsko@pgia.ru

\section{M.N. Melnik}

Polar Geophysical Institute RAS, Apatity, Russia,melnik@pgia.ru

\section{I.V. Mingalev}

Polar Geophysical Institute RAS, Apatity,Russia,mingalev_o@pgia.ru Murmansk Arctic State University, Apatity,_Russia,mingalev_i@pgia.ru

\author{
H.V. Malova \\ Skobeltsyn Institute of Nuclear Physics, \\ Lomonosov Moscow State University, \\ Moscow, Russia, hmalova@yandex.ru \\ Space Research Institute RAS, \\ Moscow, Russia, hmalova@yandex.ru
}

\section{A.M. Merzlyi}

Space Research Institute RAS, Moscow, Russia,pinega142@yandex.ru

\begin{abstract}
In this paper, we derive a divergent form of the force balance equation for collisionless plasma in the quasineutrality approximation, in which the electric field and current density are excluded. For a stationary spatially one-dimensional current sheet with a constant normal component of the magnetic field and magnetized electrons, the general form of the force balance equation has been obtained for the first time in the form of a conservation law. An equation in this form is necessary for the correct formulation of boundary conditions when modeling asymmetric current sheets, as well as for the control of the stationarity of the numerical solution obtained in the mod-
\end{abstract}

el. Furthermore, the fulfillment of this equation is considered for two types of stationary configurations of a thin current sheet, which are obtained using a numerical model. The derived equation makes it possible to develop models of asymmetric current sheets, in particular current sheets on the magnetopause flanks in the magnetotail.

Keywords: collisionless plasma, Vlasov equation, current sheet, numerical simulation, Earth's magnetosphere, magnetized electrons

\section{INTRODUCTION}

Force balance equations are needed to study properties of multiscale spatially inhomogeneous structures in collisionless space plasma, specifically to construct analytical and numerical models, as well as to analyze measurement data. An example of such structures is current sheets (CS), which play an essential role in physics of collisionless plasma of the magnetosphere and solar wind [Zelenyi et al., 2011, 2016]. They appear between the space regions where plasma parameters vary widely, and at the boundaries of regions with oppositely directed magnetic field lines. CS is commonly referred to as thin if its thickness is comparable to the gyroradius of thermal ions of the ambient plasma.

Thin current sheets (TCS) have been discovered in recent decades due to satellite studies of interplanetary and circumplanetary space of the Solar System [Zelenyi et al., 2011; McPherron et al., 1987; Sergeev et al., 1993; Runov et al., 2006; Baumjohann et al., 2007; Arons et al., 2011]. In Earth's magnetosphere, TCS constantly exists at the magnetopause and also forms in the near magnetotail during the growth phase of a magnetospheric substorm. An important feature of the latter as well as of most observed TCS is the existence of a normal magnetic field component, which makes them considerably different from sheets without the normal com- ponent [Speiser, 1965; Ashour-Abdalla et al., 1994; Zelenyi et al., 2011, 2016], with a given set of stationary analytical solutions - Harris's solution [Harris, 1962] and others [Kocharovsky et al., 2016, 2019].

TCS in the near magnetotail decays at the beginning of the substorm expansion phase, but the mechanism of its decay has not been fully elucidated and is still a hot topic of research over recent decades. To study possible mechanisms of TCS decay (e.g., owing to the development of the tearing mode [Somov et al., 1993]), it is necessary to examine in detail their stationary configurations, which for TCS with the normal component in the general case can be obtained only from numerical simulation; and in special cases, using approximate analytical models, in which the force balance equations are used to set boundary conditions and to check the correctness of the numerical solution.

In this paper, we briefly consider how force balance equations are derived for quasineutral collisionless plasma. We also define the form of one of these equations as a conservation law for a stationary spatially one-dimensional current sheet with a constant normal magnetic field component and magnetized electrons. We exemplify the use of the last equation in a numerical model of TCS.

Note that the development of numerical and approximate analytical models of stationary TCS with the normal magnetic field component has a long history, 
which is described in the reviews [Zelenyi et al., 2011, 2016] and in [Mingalev et al., 2018]. Analytical models represent protons in the quasi-adiabatic approximation [Kropotkin et al., 1995], and the most advanced models describe electrons in the so-called semi-fluid approximation (see, e.g., [Sitnov et al., 2000; Zelenyi et al., 2011]). The most advanced numerical models for protons use the particle-in-cell method to solve the stationary Vlasov equation and, as do analytical models, take into account the contribution of electrons in the semifluid approximation [Bykov et al., 2008, 2016]. These models considered symmetric TCS configurations for which boundary conditions were set from symmetry conditions, and the force balance was not addressed.

In our TCS model [Mingalev et al., 2018], the analytical description of magnetized electrons has been improved and has become totally kinetic. They are described by a Maxwell-Boltzmann distribution, which, depending on the formulation of the problem, is either an exact or approximate solution of the Vlasov equation in the drift approximation. In our model, the Vlasov equation for ions is solved numerically by the method of characteristics, thus allowing us to apply GPU-based massive parallel computations to the simulation. The findings of this work permit the use of our model for studying asymmetric TCS.

\section{FORCE BALANCE EQUATIONS IN COLLISIONLESS PLASMA}

Consider the derivation of a force balance equation a total plasma momentum flux equation in the quasineutrality approximation in which the electric field and current density are excluded and the maximum possible number of terms is given in the divergent form. This equation can be written in two forms: through current densities of plasma components or through their hydrodynamic velocities.

Denote the magnetic induction and electric field strength vectors, which depend on spatial coordinates $\mathbf{x}=\left(x_{1}, x_{2}, x_{3}\right)^{T} \in \mathbb{R}^{3}$ and time $t$, by $\mathbf{B}(\mathbf{x}, t)$ and $\mathbf{E}(\mathbf{x}, t)$ respectively. Designate the scalar and vector products of vectors $\mathbf{u}$ and $\mathbf{v}$ in space $\mathbb{R}^{3}$. as $(\mathbf{u} \cdot \mathbf{v})$ and $[\mathbf{u} \times \mathbf{v}]$ respectively. We also use the unit vector along the magnetic field $\mathbf{b}(\mathbf{x}, t)=\mathbf{B} / B$ and the electric drift velocity $\mathbf{v}_{E}(\mathbf{x}, t)=[\mathbf{E} \times \mathbf{B}] / B^{2}$, where $B=|\mathbf{B}|$. For an arbitrary vector field $\mathbf{a}(\mathbf{x}, t)$, we introduce its field-aligned component $a_{\|}=(\mathbf{a} \cdot \mathbf{b})$, and longitudinal $\mathbf{a}_{\|}=A_{\|} \mathbf{b}$ and orthogonal $\mathbf{a}_{\perp}=\mathbf{a}-\mathbf{a}_{\|}$components with respect to the magnetic field.

Assume that plasma contains $K$ ion types and electrons. Denote by $f_{\alpha}(t, \mathbf{x}, \mathbf{v})$ the distribution function of the plasma component of the type $\alpha(\alpha=1, \ldots, K$ for ion components, $\alpha=\mathrm{e}$ for electrons), which depends on $t$, $\mathbf{x} \in \mathbb{R}^{3}$, and $\mathbf{v}=\left(v_{1}, v_{2}, v_{3}\right)^{T} \in \mathbb{R}^{3}$. For particles of type $\alpha, q_{\alpha}$ and $m_{\alpha}$ imply a charge and mass of the particles; $n_{\alpha}(\mathbf{x}, t)$ and $\mathbf{j}_{\alpha}(\mathbf{x}, t)$, their concentration and current density. In this case, the proton charge $q_{1}$ is represented by $e$, i.e. for electrons $q_{e}=-e$.

In the SI system, the system of Vlasov equations along with concentration and current can be written as (1.1)-(1.3)

$$
\begin{aligned}
& \frac{\partial f_{\alpha}}{\partial t}+\left(\mathbf{v} \cdot \frac{\partial f_{\alpha}}{\partial \mathbf{x}}\right)+\frac{q_{\alpha}}{m_{\alpha}}\left((\mathbf{E}+[\mathbf{v} \times \mathbf{B}]) \cdot \frac{\partial f_{\alpha}}{\partial \mathbf{v}}\right)=0, \\
& \alpha=1, \ldots, K, \mathrm{e} \\
& n_{\alpha}(\mathbf{x}, t)=\int_{\mathbb{R}^{3}} f_{\alpha}(t, \mathbf{x}, \mathbf{v}) d^{3} \mathbf{v}, \\
& \rho_{i}(\mathbf{x}, t)=\sum_{\alpha=1}^{K} q_{\alpha} n_{\alpha}(\mathbf{x}, t), \\
& \rho=\rho_{i}-e n_{e}, \\
& \mathbf{j}_{\alpha}(\mathbf{x}, t)=q_{\alpha} \int_{\mathbb{R}^{3}} \mathbf{v} f_{\alpha}(t, \mathbf{x}, \mathbf{v}) d^{3} \mathbf{v}, \\
& \mathbf{j}_{i}(\mathbf{x}, t)=\sum_{\alpha=1}^{K} \mathbf{j}_{\alpha}(\mathbf{x}, t), \mathbf{j}=\mathbf{j}_{i}+\mathbf{j}_{e} .
\end{aligned}
$$

Hereafter, $\rho_{i}(\mathbf{x}, t)$ and $\mathbf{j}_{i}(\mathbf{x}, t)$ stand for the cumulative ion charge and current densities; $\rho(\mathbf{x}, t), \mathbf{j}(\mathbf{x}, t)$, for the total charge and current densities.

Use for each plasma component $\alpha=1, \ldots, K$, e the hydrodynamic velocity $\mathbf{u}_{\alpha}(\mathbf{x}, t)$, the stress tensor $\widehat{\boldsymbol{\Pi}}_{\alpha}(\mathbf{x}, t)$, and the pressure tensor $\widehat{\mathbf{P}}_{\alpha}(\mathbf{x}, t)$, , as well as the total stress tensor of ions $\widehat{\boldsymbol{\Pi}}_{i}(\mathbf{x}, t)$ and the total pressure tensor of ions $\widehat{\mathbf{P}}_{i}(\mathbf{x}, t)$, which are determined by formulas

$$
\begin{aligned}
& \mathbf{u}_{\alpha}(\mathbf{x}, t)=\mathbf{j}_{\alpha}(\mathbf{x}, t) /\left(q_{\alpha} n_{\alpha}(\mathbf{x}, t)\right), \\
& \widehat{\mathbf{P}}_{\alpha}(\mathbf{x}, t)= \\
& =m_{\alpha} \int_{\mathbb{R}^{3}}\left(\mathbf{v}-\mathbf{u}_{\alpha}(\mathbf{x}, t)\right) \otimes\left(\mathbf{v}-\mathbf{u}_{\alpha}(\mathbf{x}, t)\right) f_{\alpha}(t, \mathbf{x}, \mathbf{v}) d^{3} \mathbf{v}, \\
& \widehat{\mathbf{P}}_{i}=\sum_{\alpha=1}^{K} \widehat{\mathbf{P}}_{\alpha}, \\
& \widehat{\boldsymbol{\Pi}}_{\alpha}(\mathbf{x}, t)=m_{\alpha} \int_{\mathbb{R}^{3}} \mathbf{v} \otimes \mathbf{v} f_{\alpha}(t, \mathbf{x}, \mathbf{v}) d^{3} \mathbf{v}, \\
& \widehat{\boldsymbol{\Pi}}_{i}=\sum_{\alpha=1}^{K} \widehat{\boldsymbol{\Pi}}_{\alpha} .
\end{aligned}
$$

Hereafter, $\mathbf{U} \otimes \mathbf{V}$ designates the diadics formed by the vectors $\mathbf{U}$ and $\mathbf{V}$ in space $\mathbb{R}^{3}$, whose Cartesian components are determined by the respective components of its constituent vectors from Formula $(\mathbf{U} \otimes \mathbf{V})_{k, l}=U_{k} V_{l}$. Note that Formula

$$
\widehat{\boldsymbol{\Pi}}_{\alpha}=\widehat{\mathbf{P}}_{\alpha}+m_{\alpha} n_{\alpha} \mathbf{u}_{\alpha} \otimes \mathbf{u}_{\alpha},
$$

is valid, which represents the decomposition of the stress tensor $\widehat{\boldsymbol{\Pi}}_{\alpha}$ into the sum of the pressure tensor $\widehat{\mathbf{P}}_{\alpha}$ and the inertia tensor $m_{\alpha} n_{\alpha} \mathbf{u}_{\alpha} \otimes \mathbf{u}_{\alpha}$.

Vlasov equations (1.1) for each plasma component $\alpha=1, \ldots, K$, e yield a momentum flux equation, which can be represented in two forms: as the current density

$$
\frac{\partial \mathbf{j}_{\alpha}}{\partial t}=\frac{q_{\alpha}{ }^{2} n_{\alpha}}{m_{\alpha}} \mathbf{E}-\frac{q_{\alpha}}{m_{\alpha}}\left[\mathbf{B} \times \mathbf{j}_{\alpha}\right]-\frac{q_{\alpha}}{m_{\alpha}} \operatorname{div} \widehat{\boldsymbol{\Pi}}_{\alpha},
$$

and as the hydrodynamic velocity 


$$
m_{\alpha} n_{\alpha} \frac{d_{\alpha} \mathbf{u}_{\alpha}}{d t}=q_{\alpha} n_{\alpha} \mathbf{E}-\left[\mathbf{B} \times \mathbf{j}_{\alpha}\right]-\operatorname{div} \widehat{\mathbf{P}}_{\alpha}
$$

where the traditional designation is used for the total time derivative of the arbitrary function $\Phi(\mathbf{x}, t)$ along current lines of each plasma component:

$$
\frac{d_{\alpha} \Phi(\mathbf{x}, t)}{d t}=\frac{\partial \Phi(\mathbf{x}, t)}{\partial t}+\left(\mathbf{u}_{\alpha}(\mathbf{x}, t) \cdot \nabla\right) \Phi(\mathbf{x}, t) .
$$

Derive two divergent forms of the force balance equation, which follow from the momentum flux equation in the form of (1.7) and (1.8) respectively. The summation of the momentum flux equations in the form of (1.7), multiplied by the ratio $m_{\alpha} / q_{\alpha}$, over all plasma components, and then the consideration of determinations of the charge density in (1.2), the total current in (1.3), and the total stress tensor of ions in (1.5) give

$$
\begin{aligned}
& -\frac{m_{\mathrm{e}}}{e} \frac{\partial \mathbf{j}_{\mathrm{e}}}{\partial t}+\sum_{\alpha=1}^{K} \frac{m_{\alpha}}{q_{\alpha}} \frac{\partial \mathbf{j}_{\alpha}}{\partial t}= \\
& =\rho \mathbf{E}-[\mathbf{B} \times \mathbf{j}]-\operatorname{div} \widehat{\boldsymbol{\Pi}}_{\mathrm{i}}-\operatorname{div} \widehat{\boldsymbol{\Pi}}_{\mathrm{e}}
\end{aligned}
$$

Similarly, the summation of the momentum flux equations in the form of (1.8) for all plasma components $\alpha=1, \ldots, K$, e in view of determining the charge density and the ion current in (1.2) and (1.3), as well as the total pressure tensor of ions in (1.4) results in

$$
\begin{aligned}
& m_{\mathrm{e}} n_{\mathrm{e}} \frac{d_{\mathrm{e}} \mathbf{u}_{\mathrm{e}}}{d t}+\sum_{\alpha=1}^{K} m_{\alpha} n_{\alpha} \frac{d_{\alpha} \mathbf{u}_{\alpha}}{d t}= \\
& =\rho \mathbf{E}-[\mathbf{B} \times \mathbf{j}]-\operatorname{div} \widehat{\mathbf{P}}_{\mathrm{i}}-\operatorname{div} \widehat{\mathbf{P}}_{\mathrm{e}}
\end{aligned}
$$

The quasineutrality condition $\rho(\mathbf{x}, t) \equiv 0$ and resulting biasing equality to zero allow us to eliminate the electric field from these equations, and to express the current density as $\mathbf{j}=\operatorname{rot} \mathbf{B} / \mu_{0}$ from the Ampere equation. As a result, in view of the representation of the term $[\mathbf{B} \times \operatorname{rot} \mathbf{B}]$ in the divergent form

$$
[\mathbf{B} \times \operatorname{rot} \mathbf{B}]=\operatorname{div}\left(\frac{1}{2} B^{2} \hat{\mathbf{I}}-\mathbf{B} \otimes \mathbf{B}\right),
$$

( $\hat{\mathbf{I}}$ is the unit tensor), Equations (1.10) and (1.11) yield force balance equations in divergent forms

$$
\begin{aligned}
& -\frac{m_{\mathrm{e}}}{e} \frac{\partial \mathbf{j}_{\mathrm{e}}}{\partial t}+\sum_{\alpha=1}^{K} \frac{m_{\alpha}}{q_{\alpha}} \frac{\partial \mathbf{j}_{\alpha}}{\partial t}= \\
& =\operatorname{div}\left(\frac{1}{\mu_{0}} \mathbf{B} \otimes \mathbf{B}-\frac{1}{2 \mu_{0}} B^{2} \widehat{\mathbf{I}}-\widehat{\boldsymbol{\Pi}}_{\mathrm{i}}-\widehat{\boldsymbol{\Pi}}_{\mathrm{e}}\right), \\
& m_{\mathrm{e}} n_{\mathrm{e}} \frac{d_{\mathrm{e}} \mathbf{u}_{\mathrm{e}}}{d t}+\sum_{\alpha=1}^{K} m_{\alpha} n_{\alpha} \frac{d_{\alpha} \mathbf{u}_{\alpha}}{d t}= \\
& =\operatorname{div}\left(\frac{1}{\mu_{0}} \mathbf{B} \otimes \mathbf{B}-\frac{1}{2 \mu_{0}} B^{2} \hat{\mathbf{I}}-\widehat{\mathbf{P}}_{\mathrm{i}}-\widehat{\mathbf{P}}_{\mathrm{e}}\right),
\end{aligned}
$$

which are the main result of this section.

Note that on the right side of Equation (1.12) is the difference between the bulk density of magnetic tension force $\frac{1}{\mu_{0}}(\mathbf{B} \cdot \nabla) \mathbf{B}=\frac{1}{\mu_{0}} \operatorname{div}(\mathbf{B} \otimes \mathbf{B})$ and the divergence of the sum of the magnetic pressure ten$\operatorname{sor}\left(\mathbf{B}^{2} /\left(2 \mu_{0}\right)\right) \hat{\mathbf{I}}$, the total stress tensor of ions, $\widehat{\boldsymbol{\Pi}}_{\mathrm{i}}$ and the stress tensor of electrons $\widehat{\boldsymbol{\Pi}}_{\mathrm{e}}$. An imbalance between these forces violates the stationarity of the process. A similar imbalance on the right side of Equation (1.13) implies the appearance of the bulk density of the force acting on the plasma.

In [Mingalev et al., 2020], we have shown that in the case of collisionless plasma consisting of nonmagnetized ions and magnetized electrons, the electrons are in full force balance:

$$
\frac{d_{\mathrm{e}} \mathbf{u}_{\mathrm{e}}}{d t}=0 \Leftrightarrow \frac{\partial \mathbf{j}_{\mathrm{e}}}{\partial t}=e \operatorname{div}\left(n_{\mathrm{e}} \mathbf{u}_{\mathrm{e}} \otimes \mathbf{u}_{\mathrm{e}}\right) .
$$

Substituting this condition into (1.12) reduces it to the form

$$
\begin{aligned}
& \sum_{\alpha=1}^{K} \frac{m_{\alpha}}{q_{\alpha}} \frac{\partial \mathbf{j}_{\alpha}}{\partial t}= \\
& =\operatorname{div}\left(\frac{1}{\mu_{0}} \mathbf{B} \otimes \mathbf{B}-\frac{1}{2 \mu_{0}} B^{2} \hat{\mathbf{I}}-\widehat{\boldsymbol{\Pi}}_{\mathrm{i}}-\widehat{\mathbf{P}}_{\mathrm{e}}\right),
\end{aligned}
$$

where, as compared to (1.12), on the left side the term with the time derivative of the electron current cancels out, and on the right side the electron stress tensor $\widehat{\boldsymbol{\Pi}}_{\mathrm{e}}$ is replaced by their pressure tensor $\widehat{\mathbf{P}}_{\mathrm{e}}$, which is defined by

$$
\widehat{\mathbf{P}}_{\mathrm{e}}=p_{\mathrm{e} \perp} \hat{\mathbf{I}}+\left(p_{\mathrm{e} \|}-p_{\mathrm{e} \perp}\right) \mathbf{b} \otimes \mathbf{b},
$$

where $p_{\mathrm{e} \|}$ and $p_{\mathrm{e} \perp}$ are field-aligned and transverse pressure of electrons. Substituting (1.14) into (1.13) reduces it to the form

$$
\begin{aligned}
& \sum_{\alpha=1}^{K} m_{\alpha} n_{\alpha} \frac{d_{\alpha} \mathbf{u}_{\alpha}}{d t}= \\
& =\operatorname{div}\left(\frac{1}{\mu_{0}} \mathbf{B} \otimes \mathbf{B}-\frac{1}{2 \mu_{0}} B^{2} \hat{\mathbf{I}}-\widehat{\mathbf{P}}_{\mathrm{i}}-\widehat{\mathbf{P}}_{\mathrm{e}}\right),
\end{aligned}
$$

where, as compared to (1.13), on the left side the inertial term of electrons cancels out.

Compared to Equation (1.12), the physical meaning of Equation (1.15) has slightly changed. On its right side is the difference between the bulk density of magnetic tension force $\frac{1}{\mu_{0}}(\mathbf{B} \cdot \nabla) \mathbf{B}=\frac{1}{\mu_{0}} \operatorname{div}(\mathbf{B} \otimes \mathbf{B})$ and the divergence of the sum of the magnetic pressure tensor $\left(\mathbf{B}^{2} /\left(2 \mu_{0}\right)\right) \hat{\mathbf{I}}$, the total stress tensor of ions $\widehat{\boldsymbol{\Pi}}_{\mathrm{i}}=\sum_{\alpha=1}^{K} \widehat{\boldsymbol{\Pi}}_{\alpha}$, and the pressure tensor of electrons $\widehat{\mathbf{P}}_{\mathrm{e}}$. Equations (1.15) are suitable for setting out the boundary conditions in models of stationary asymmetric current sheets.

Note that in the case of collisionless plasma consisting of protons and magnetized electrons, force balance equations (1.15) and (1.17) take the form 


$$
\begin{aligned}
& \frac{m_{\mathrm{p}}}{e} \frac{\partial \mathbf{j}_{\mathrm{p}}}{\partial t}= \\
& =\operatorname{div}\left(\frac{1}{\mu_{0}} \mathbf{B} \otimes \mathbf{B}-\frac{1}{2 \mu_{0}} B^{2} \hat{\mathbf{I}}-\widehat{\boldsymbol{\Pi}}_{\mathrm{p}}-\widehat{\mathbf{P}}_{\mathrm{e}}\right), \\
& m_{\mathrm{p}} n_{\mathrm{p}} \frac{d_{\mathrm{p}} \mathbf{u}_{\mathrm{p}}}{d t}= \\
& =\operatorname{div}\left(\frac{1}{\mu_{0}} \mathbf{B} \otimes \mathbf{B}-\frac{1}{2 \mu_{0}} B^{2} \hat{\mathbf{I}}-\widehat{\mathbf{P}}_{\mathrm{p}}-\widehat{\mathbf{P}}_{\mathrm{e}}\right),
\end{aligned}
$$

and are divergent forms of the momentum flux equation (motion equation) for protons in which the electric field and current density are omitted.

\section{FORCE BALANCE EQUATIONS IN ONE-DIMENSIONAL CURRENT SHEET}

Consider force balance equations (1.12) and (1.15) for a stationary spatially one-dimensional current sheet in which all functions depend only on one spatial coordinate $z$ across the layer. Depending on the situation, we denote the vectors of the Cartesian coordinate system by $\mathbf{e}_{x}, \mathbf{e}_{y}$, and $\mathbf{e}_{z}$, as well as the coordinate vector components $\mathbf{x}=x \mathbf{e}_{x}+y \mathbf{e}_{y}+z \mathbf{e}_{z} \in \mathbb{R}^{3}$ and the velocity vector components $\mathbf{v}=v_{x} \mathbf{e}_{x}+v_{y} \mathbf{e}_{y}+v_{z} \mathbf{e}_{z} \in \mathbb{R}^{3}$.

In the CS of interest, the magnetic field has a given constant normal component $B_{z} \equiv$ const and selfconsistent components $B_{x}(z)$ and $B_{y}(z)$, and the electric field has one self-consistent component $E_{z}(z)$ :

$$
\begin{aligned}
& \mathbf{B}(z)=B_{x}(z) \mathbf{e}_{x}+B_{y}(z) \mathbf{e}_{y}+B_{z} \mathbf{e}_{z}, \\
& \mathbf{E}(z)=E_{z}(z) \mathbf{e}_{z}=-\frac{d \varphi(z)}{d z} \mathbf{e}_{z},
\end{aligned}
$$

where $\varphi(z)$ signifies a scalar potential. In this case, (1.12) yields a vector force balance equation in the form of a conservation law for a spatially one-dimensional current sheet

$$
\begin{aligned}
& \left(\boldsymbol{\Pi}_{i, x, z}+\boldsymbol{\Pi}_{e, \chi, z}\right) \mathbf{e}_{x}+\left(\boldsymbol{\Pi}_{i, y, z}+\boldsymbol{\Pi}_{e, y, z}\right) \mathbf{e}_{y}+ \\
& +\left(\boldsymbol{\Pi}_{i, z, z}+\boldsymbol{\Pi}_{e, z, z}+\frac{B^{2}}{2 \mu_{0}}\right) \mathbf{e}_{z}-\frac{B_{z} \mathbf{B}}{\mu_{0}} \equiv \text { const. }
\end{aligned}
$$

The equation in this form is needed to derive boundary conditions in numerical models of thin current sheets and to determine the stationarity of the resulting CS configuration.

Similarly, from stationary equation (1.15) follows a vector force balance equation in the form of a conservation law for a stationary spatially one-dimensional current sheet

$$
\begin{aligned}
& \boldsymbol{\Pi}_{i, x, z} \mathbf{e}_{x}+\boldsymbol{\Pi}_{i, y, z} \mathbf{e}_{y}+\left(\boldsymbol{\Pi}_{i, z, z}+\frac{B^{2}}{2 \mu_{0}}+p_{\mathrm{e} \perp}\right) \mathbf{e}_{z}+ \\
& +\frac{B_{z} \mathbf{B}}{\mu_{0}}\left(\frac{\mu_{0}}{B^{2}}\left(p_{\mathrm{e} \|}-p_{\mathrm{e} \perp}\right)-1\right) \equiv \text { const. }
\end{aligned}
$$

For isotropic electrons $p_{\mathrm{e} \|}(z) \equiv p_{\mathrm{e} \perp}(z) \equiv p(z)$, they contribute only to the balance along the Z-axis orthogonal to the sheet, and Equation (2.3) takes the form:

$$
\begin{aligned}
& \boldsymbol{\Pi}_{i, x, z} \mathbf{e}_{x}+\boldsymbol{\Pi}_{i, y, z} \mathbf{e}_{y}+ \\
& +\left(\boldsymbol{\Pi}_{i, z, z}+\frac{B^{2}}{2 \mu_{0}}+p_{\mathrm{e}}\right) \mathbf{e}_{z}-\frac{B_{z} \mathbf{B}}{\mu_{0}} \equiv \text { const. }
\end{aligned}
$$

This equation gives scalar force balance equations for each of the coordinate axes $\mathrm{X}, \mathrm{Y}$, and $\mathrm{Z}$ respectively,

$$
\begin{aligned}
& \Pi_{i, x, z}(z)-\frac{1}{\mu_{0}} B_{z} B_{x}(z) \equiv \text { const }, \\
& \Pi_{i, y, z}(z)-\frac{1}{\mu_{0}} B_{z} B_{y}(z) \equiv \text { const, } \\
& \Pi_{i, z, z}(z)+\frac{1}{2 \mu_{0}} B^{2}(z)+p_{\mathrm{e}}(z) \equiv \text { const. }
\end{aligned}
$$

\section{EQUATIONS OF NUMERICAL MODEL OF SPATIALLY ONE-DIMENSIONAL TCS}

Consider the most general version of the model of stationary spatially one-dimensional current sheet with magnetized electrons, in which the magnetic field has two self-consistent components and one constant normal component and which allows asymmetric configurations as well as a plasma flow through the sheet.

This model version is a generalization of the initial version [Mingalev et al., 2018], in which the magnetic field has only one self-consistent shear symmetric component.

The model has a dimension of 1D3V and makes the following assumptions.

1. All functions depend only on one spatial coordinate $z$ across the sheet; the simulation region is a strip $\{|z|<L\}$.

2. The magnetic and electric fields are given by (2.1), above and below the region $\{|z|<L\}$ the magnetic field is assumed to be constant; and the electric field, zero.

3 . The sheet is supported by field-aligned counter ion flows. The distribution function $f_{\alpha}^{( \pm)}(z, \mathbf{v})$ of ions of this type in incident plasma flows at the boundary of the calculation domain has the form of a shifted Maxwellian distribution with a hydrodynamic mean speed $\mathbf{U}_{\alpha}^{( \pm)}=-\frac{z \mathbf{B}^{( \pm)}}{|z|\left|\mathbf{B}^{( \pm)}\right|} V_{D \alpha}^{( \pm)}$, which has a value $V_{D \alpha}^{( \pm)}$and is directed along magnetic field lines toward the sheet,

$$
\begin{aligned}
& f_{\alpha}^{( \pm)}(\mathbf{v})= \\
& =\frac{n_{\alpha}^{( \pm)}}{\left(V_{\mathrm{T} \alpha}^{( \pm)} \sqrt{2 \pi}\right)^{3}} \exp \left(-\frac{1}{2\left(V_{\mathrm{T} \alpha}^{( \pm)}\right)^{2}}\left|\mathbf{v}-\mathbf{U}_{\alpha}^{( \pm)}\right|^{2}\right), \\
& \frac{Z}{|Z|}\left(\mathbf{B}^{( \pm)} \cdot \mathbf{v}\right)<0 .
\end{aligned}
$$


Here, $n_{\alpha}^{( \pm)}$is the density, $T_{\alpha}^{( \pm)}$is the temperature in electron volts, $V_{\mathrm{T} \alpha}^{( \pm)}=\sqrt{e T_{\alpha}^{( \pm)} / m_{\alpha}}$ is the thermal velocity in these flows. The superscript $(+)$ indicates a downflow; and (-), an upflow.

In the model, the ion components are described by stationary Vlasov equations, which are solved numerically. Magnetized electrons are described by a stationary Vlasov equation in the drift approximation (see [Rudakov, Sagdeev, 1958; Morozov, 1963; Volkov, 1964; Kalsrud, 1983; Ilgisonis, 1993; Mingalev et al., 2020]), and their current density, according to the drift theory, is given by Formula

$$
\begin{aligned}
& \mathbf{j}_{\mathrm{e}}(z)=j_{\mathrm{e} \|}(z) \mathbf{b}(z)-e n_{\mathrm{e}} \mathbf{v}_{E}+ \\
& +\left(p_{\mathrm{e} \|}-p_{\mathrm{e} \perp}\right) \frac{[\mathbf{b} \times(\mathbf{b} \cdot \nabla) \mathbf{b}]}{B}+\frac{\left[\mathbf{b} \times \nabla p_{\mathrm{e} \perp}\right]}{B},
\end{aligned}
$$

The electric field is determined from the condition of the field-aligned force balance of electrons

$$
\begin{aligned}
& E_{\|}=(\mathbf{b} \cdot \mathbf{E})=-\frac{1}{e n_{e}}\left(\mathbf{b} \cdot \operatorname{div} \widehat{\mathbf{P}}_{\mathrm{e}}\right)= \\
& =\frac{1}{e n_{\mathrm{e}}}\left(\left(p_{\mathrm{e} \|}-p_{\mathrm{e} \perp}\right)(\mathbf{b} \cdot \nabla \ln B)-\left(\mathbf{b} \cdot \nabla p_{\mathrm{e} \|}\right)\right),
\end{aligned}
$$

which in this case takes the form

$$
E_{z}=\frac{1}{\rho_{\mathrm{i}}}\left(-\frac{d p_{\mathrm{e} \|}}{d z}+\frac{\left(p_{\mathrm{e} \|}-p_{\mathrm{e} \perp}\right)}{B} \frac{d B}{d z}\right) .
$$

Fulfillment of the condition $\operatorname{div} \mathbf{j}=0$ in the problem with fields of type (2.1) is equivalent to the absence of the $z$ component in the total current density $j_{z}(z) \equiv 0$, , which is provided by the neutralizing field-aligned electron current, i.e. the total current density is defined by

$$
\begin{aligned}
& \mathbf{j}(z)=\mathbf{j}_{\mathrm{i}}(z)+\mathbf{j}_{\mathrm{e}}(z)= \\
& =\mathbf{j}_{\mathrm{i}}(z)+\mathbf{j}_{\mathrm{e} \perp}(z)+j_{\mathrm{e} \|}(z) \mathbf{b}(z),
\end{aligned}
$$

where the ion current density $\mathbf{j}_{\mathbf{i}}(z)$ is computed numerically. Substituting (3.5) into the equality $j_{z}(z) \equiv 0$ allows us to derive the field-aligned electron current density component

$$
\begin{aligned}
& j_{\mathrm{e} \|}(z)=-\frac{\left(j_{\mathrm{iz}}(z)+j_{\mathrm{e} \perp z}(z)\right)}{\left(\mathbf{b}(z) \cdot \mathbf{e}_{z}\right)}= \\
& =-\frac{B(z)}{B_{z}}\left(j_{\mathrm{iz}}(z)+j_{\mathrm{e} \perp z}(z)\right),
\end{aligned}
$$

which gives the following formulas for the electron current density and the total current density:

$$
\begin{aligned}
& \mathbf{j}_{\mathrm{e}}(z)=\mathbf{j}_{\mathrm{e} \perp}(z)-\left(j_{\mathrm{i} z}(z)+j_{\mathrm{e} \perp z}(z)\right) \frac{\mathbf{B}(z)}{B_{z}} \\
& \mathbf{j}(z)=\mathbf{j}_{\mathrm{i}}(z)+\mathbf{j}_{\mathrm{e} \perp}(z)-\left(j_{\mathrm{i} z}(z)+j_{\mathrm{e} \perp z}(z)\right) \frac{\mathbf{B}(z)}{B_{z}} .
\end{aligned}
$$

Thus, the Ampere equation $\operatorname{rot} \mathbf{B}=\mu_{0} \mathbf{j}$ in the model reduces to a system of two first-order ordinary differential equations relative to the self-consistent magnetic field components

$$
\frac{d B_{x}(z)}{d z}=\mu_{0} j_{y}(z), \frac{d B_{y}(z)}{d z}=-\mu_{0} j_{x}(z),
$$

in which the right side may depend on $B_{x}(z)$ and $B_{y}(z)$, as well as on their derivatives.

Substituting Formulas (3.6) and (3.4) in (3.2), in view of (2.1), allows us to represent the electron current density as

$$
\mathbf{j}_{\mathrm{e}}(z)=-j_{\mathrm{iz} z} \frac{\mathbf{B}}{B_{z}}-\frac{d}{d z}\left(\frac{\left(p_{\mathrm{e} \|}-p_{\mathrm{e} \perp}\right)}{B^{2}}\left[\mathbf{B} \times \mathbf{e}_{z}\right]\right) .
$$

From this formula it follows that for isotropic electrons $p_{\mathrm{e} \|}=p_{\mathrm{e} \perp}=p_{\mathrm{e}}$ they can give only a neutralizing fieldaligned current

$$
\begin{aligned}
& \mathbf{j}_{\mathrm{e}}(z)=-j_{\mathrm{iz}}(z) \frac{\mathbf{B}(z)}{B_{z}}, \\
& \mathbf{j}(z)=\mathbf{j}_{\mathrm{i}}(z)-j_{\mathrm{i} z}(z) \frac{\mathbf{B}(z)}{B_{z}} .
\end{aligned}
$$

For the Vlasov equation describing magnetized electrons in the drift approximation the characteristic system is a system of guiding center motion equations (see [Rudakov, Sagdeev, 1958; Morozov, 1963; Volkov, 1964; Kalsrud, 1983; Ilgisonis, 1993; Mingalev et al., 2020]). For this system of equations according to the drift theory, the magnetic moment $\mu\left(z, v_{L}\right)$ and the total energy (Hamiltonian) $H\left(z, v_{\|}, v_{L}\right)$, which are defined by

$$
\begin{aligned}
& \mu\left(z, v_{L}^{2}\right)=\frac{v_{L}^{2}}{2 B(z)}, \\
& H\left(z, v_{\|}^{2}, v_{L}^{2}\right)=\frac{m_{\mathrm{e}}}{2}\left(v_{\|}{ }^{2}+v_{L}^{2}\right)-e \varphi(z),
\end{aligned}
$$

are approximate independent integrals. If the magnetic field of type (2.1) has no shear component $B_{y}(z) \equiv 0$, the functions $\mu\left(z, v_{L}\right)$ and $H\left(z, v_{\|}, v_{L}\right)$ are exact integrals. In this case, the general solution of the stationary Vlasov equation in the drift approximation for electrons has the form of an arbitrary function of these two integrals:

$$
\begin{aligned}
& F_{\mathrm{e}}\left(z, v_{\|}, v_{L}\right)= \\
& =\Phi\left(\mu\left(z, v_{L}^{2}\right), H\left(z, v_{\|}^{2}, v_{L}^{2}\right)\right),
\end{aligned}
$$

where $\Phi(\mu, H)$ is a sufficiently smooth function of two variables. If the shear magnetic field component $B_{y}(z) \neq 0$, the function of type (3.12) is an approximate solution. The simplest variant is the special case when electrons in a current sheet and outside it have the Maxwell-Boltzmann distribution in the stationary magnetic and electric field, i.e. the distribution function of their guiding centers may be represented as 


$$
\begin{aligned}
& F_{\mathrm{e}}\left(z, v_{\|}, v_{L}\right)=\frac{\left(1+\gamma_{0}\right) n_{0}}{\left(V_{\mathrm{Te} 0} \sqrt{2 \pi}\right)^{3}} \exp \left(\frac{\varphi(z)}{T_{\mathrm{e} 0}}\right) \times \\
& \times \exp \left(-\frac{v_{\|}^{2}}{2 V_{\mathrm{Te} 0}^{2}}\right) \exp \left(-\frac{v_{L}^{2}}{2 V_{\mathrm{Te} 0}^{2}}\left(1+\frac{\gamma_{0} B_{0}}{B(z)}\right)\right),
\end{aligned}
$$

where the constants

$$
\begin{aligned}
& B_{0}=B\left(z_{0}\right), n_{0}=n\left(z_{0}\right), \\
& \gamma_{0}=\gamma\left(z_{0}\right)=\frac{p_{\mathrm{e} \|}\left(z_{0}\right)-p_{\mathrm{e} \perp}\left(z_{0}\right)}{p_{\mathrm{e} \perp}\left(z_{0}\right)},
\end{aligned}
$$

are values of the respective functions at a point $z_{0}$ of the sheet, i.e. $\gamma_{0}$ is the dimensionless anisotropy parameter $\left(\gamma_{0}=0\right.$ in the isotropic case), $T_{\mathrm{e} 0}$ is the electron temperature in electron volts at this point, and $V_{\mathrm{Te} 0}=\sqrt{e T_{\mathrm{e} 0} / m_{\mathrm{e}}}$ is the corresponding electron thermal velocity. Distribution function (3.13) gives the following expression for the electron density in terms of the scalar potential and magnetic field:

$$
\begin{aligned}
& n_{\mathrm{e}}(z)=n_{\mathrm{i}}(z)=n(z)= \\
& =\frac{\left(1+\gamma_{0}\right) n_{0} B(z)}{\left(B(z)+\gamma_{0} B_{0}\right)} \exp \left(\frac{\varphi(z)}{T_{\mathrm{e} 0}}\right),
\end{aligned}
$$

which expresses the potential through the electron density and the magnetic field:

$$
\varphi(z)=T_{\mathrm{e} 0} \ln \left(\frac{\left(B(z)+\gamma_{0} B_{0}\right)}{\left(1+\gamma_{0}\right) B(z)} \frac{n(z)}{n_{0}}\right) .
$$

Substituting (3.14) in the formula for the longitudinal pressure yields

$$
p_{\mathrm{e} \|}(z)=e n(z) T_{\mathrm{e} 0} .
$$

The first expression suggests that the longitudinal electron temperature component is constant in the sheet:

$$
T_{\mathrm{e} \|}(z)=\frac{p_{\mathrm{e} \|}(z)}{e n_{\mathrm{e}}(z)}=\frac{e n(z) T_{\mathrm{e} 0}}{e n(z)}=T_{\mathrm{e} 0} \equiv \text { const } .
$$

Hereinafter, the longitudinal temperature is therefore considered constant $T_{\mathrm{e} \|}=T_{\mathrm{e} 0} \equiv$ const .

Distribution function (3.13) also gives the following formula for the lateral pressure:

$$
\begin{aligned}
& p_{\mathrm{e} \perp}(z)= \\
& =e T_{\mathrm{e} \|} n_{0} \exp \left(\frac{\varphi(z)}{T_{\mathrm{e} \|}}\right)\left(\frac{B(z)}{\left(B(z)+\gamma_{0} B_{0}\right)}\right)^{2}= \\
& =\frac{e n(z) T_{\mathrm{e} \|} B(z)}{\left(B(z)+\gamma_{0} B_{0}\right)}=\frac{p_{\mathrm{e} \|}(z) B(z)}{\left(B(z)+\gamma_{0} B_{0}\right)} .
\end{aligned}
$$

From this formula follows an expression for the orthogonal electron temperature in the sheet

$$
T_{\mathrm{e} \perp}(z)=\frac{p_{\mathrm{e} \perp}(z)}{e n(z)}=\frac{T_{\mathrm{e} \|} B(z)}{\left(B(z)+\gamma_{0} B_{0}\right)},
$$

and for the electron pressure difference:

$$
\begin{aligned}
& p_{\mathrm{e} \|}(z)-p_{\mathrm{e} \perp}(z)=\frac{\gamma_{0} B_{0} p_{\mathrm{e} \|}(z)}{\left(B(z)+\gamma_{0} B_{0}\right)}= \\
& =\frac{e T_{\mathrm{e} \|} \gamma_{0} B_{0} n(z)}{\left(B(z)+\gamma_{0} B_{0}\right)} .
\end{aligned}
$$

Substituting this expression into (3.9) and then substituting the result into (3.7) lead to the following expressions for current density components

$$
\begin{aligned}
& j_{x}(z)=j_{\mathrm{ix}}(z)-j_{\mathrm{iz}}(z) \frac{B_{x}(z)}{B_{z}}- \\
& -e T_{\mathrm{e} \|} \gamma_{0} B_{0} \frac{d}{d z}\left(\frac{B_{y}(z) n(z)}{\left(B(z)+\gamma_{0} B_{0}\right) B^{2}(z)}\right), \\
& j_{y}(z)=j_{\mathrm{iy}}(z)-j_{\mathrm{iz}}(z) \frac{B_{y}(z)}{B_{z}}+ \\
& +e T_{\mathrm{e} \|} \gamma_{0} B_{0} \frac{d}{d z}\left(\frac{B_{x}(z) n(z)}{\left(B(z)+\gamma_{0} B_{0}\right) B^{2}(z)}\right) .
\end{aligned}
$$

Note that Formulas (3.18), (3.19) suggest that if electrons are isotropic outside of the current sheet $(\gamma$ ${ }_{0}=0$ ), they are isotropic inside the sheet as well, their temperature is constant: $T_{\mathrm{e}} \equiv$ const , and their current density and the total current density are defined by Formulas (3.10). In this case, Formulas (3.14)-(3.19) take the following form:

$$
\begin{aligned}
& F_{\mathrm{e}}\left(z, v_{\|}, v_{L}\right)= \\
& =\frac{n_{0}}{\left(V_{\mathrm{Te}} \sqrt{2 \pi}\right)^{3}} \exp \left(\frac{\varphi(z)}{T_{\mathrm{e}}}\right) \exp \left(-\frac{v_{\|}^{2}+v_{L}^{2}}{2 V_{\mathrm{Te}}^{2}}\right), \\
& \varphi(z)=T_{\mathrm{e}} \ln \left(\frac{n(z)}{n_{0}}\right), p_{\mathrm{e}}(z)=\operatorname{en}(z) T_{\mathrm{e}}
\end{aligned}
$$

\section{EXAMPLES OF FORCE BALANCE IN A THIN CURRENT SHEET}

As an example, consider two types of stationary configurations of spatially one-dimensional TCS with a given constant normal magnetic field component, in which there is only one ion component - protons and electrons are isotropic, i.e. the orthogonal part of their current density, in view of (3.9), is absent: $\mathbf{j}_{\mathrm{e} \perp}(z) \equiv 0$.

The main features of the technique for numerical solution of the stationary Vlasov equation are discussed in detail in [Mingalev et al., 2018]. In this case, in the course of the numerical solution of the Vlasov equation for protons, we calculated their density $n(z)=n_{i}(z)$, the current density, and the stress tensor components defined in (1.5). The electric field and the pressure were calculated by Formulas (3.24).

Input parameters were taken typical for CS of the near tail of Earth's magnetosphere in the substorm growth phase [Sergeev et al., 1993, 1996; Runov et al., 2006; Baumjohann et al., 2007; Artemyev, Zelenyi, 2013; Artemyev et al., 2013; Frank et al., 2016; Malova et al., 2017]. The halfwidth of the simulation region 
$L=R_{\mathrm{E}}=6400 \mathrm{~km}$, the spatial grid spacing $\Delta \mathrm{z}=R_{\mathrm{E}} / 640=10$ $\mathrm{km}$. The electron temperature was assumed to be $T_{\mathrm{e}}=0.5$ $\mathrm{keV}$. The temperature of protons in flows forming CS was the same above and below, $T_{\mathrm{p} 0}=4 \mathrm{keV}$, which yielded a thermal velocity value $V_{\mathrm{Tp} 0}=\sqrt{e T_{\mathrm{p} 0} / m_{\mathrm{p}}} \approx 619 \mathrm{~km} / \mathrm{s}$. The hydrodynamic velocity in these flows was deemed equal on both sides ( $V_{D}^{( \pm)}=V_{D}$ ) and was given by $V_{D}=\delta V_{\mathrm{Tp}} 0$; a set of values of the dimensionless parameter $\delta$ was also considered. The value of the magnetic field $x$ component above and below the sheet was assumed to be equal: $B_{x 0}^{(+)}=B_{x 0}^{(-)}=B_{x 0}=20 \mathrm{nT}$, and the magnetic field $z$ component normal to the sheet $B_{z}=B_{x 0 / 2}=10 \mathrm{nT}$.

\subsection{Symmetric configurations without mag- netic field shear}

Configurations of this type are best known and have been extensively studied using analytical and numerical models [Zelenyi et al., 2011, 2016; Mingalev et al., 2018]. Figure 1 exemplifies the configuration of the first type for which the magnetic field in the sheet has two components - self-consistent $B_{x}(z)$ and constant $B_{z}-$ and is symmetric $B_{x}(-z) \equiv-B_{x}(z)$. The electron current density is equal to zero: $\mathbf{j}_{\mathrm{e}}(z) \equiv 0$, and the total current density has only the $y$ component $j_{y}(z)=j_{\mathrm{iy}}(z)$.

Figure 2 shows a force balance in nanopaskal for the TCS configuration in Figure 1. Panel $a$ demonstrates the balance along the X-axis, which is defined by Equation (2.5). The force balance is seen to be fulfilled with good accuracy, and the constant on the right side of Formula (2.5) is equal to zero. Panel $b$ shows the balance along the $\mathrm{Y}$-axis, which is defined by Equation (2.6). In the

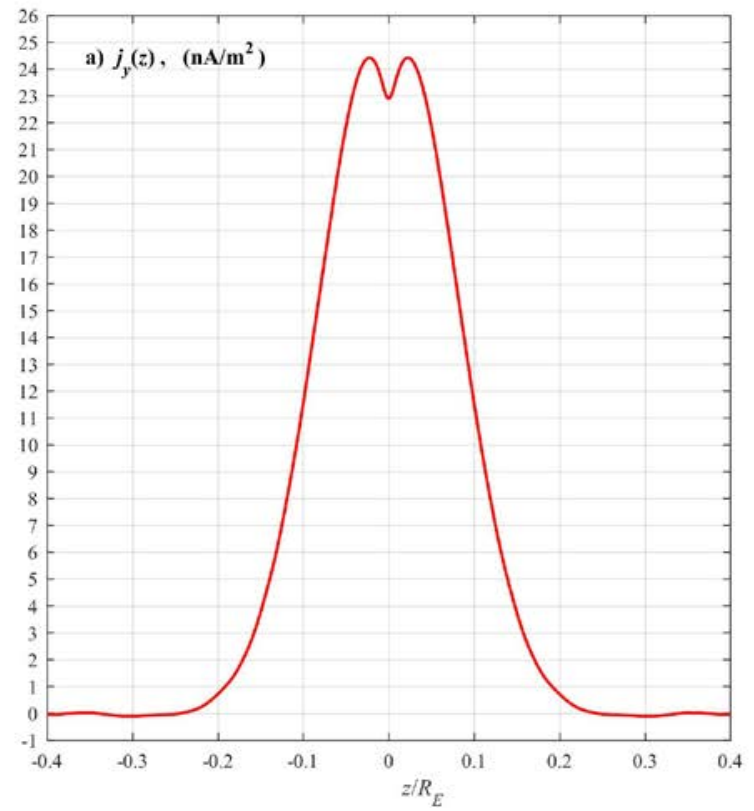

case considered, $B_{y} \equiv 0$ and the constant on the right side of Formula (2.6) is also zero. Panel $c$ depicts the balance along the Z-axis, which is defined by Equation (2.7). In view of $B_{y} \equiv 0$ and $B_{z} \equiv$ const, this equation can be written as

$$
\Pi_{i, z, z}(z)+\frac{1}{2 \mu_{0}}\left|B_{x}(z)\right|^{2}+p_{\mathrm{e}}(z) \equiv \text { const } .
$$

The red line indicates that this equation is satisfied with very high accuracy. The electron pressure $p_{\mathrm{e}}(z)$ (brown line) is seen to make though a relatively small but very important contribution to the precise fulfillment of the force balance equation in the current sheet because it compensates the sum (green line) of the proton stress tensor and the magnetic pressure for the local minimum in the center of the sheet. For other values of the parameter $\delta$, the force balance is fulfilled in a similar way.

Thus, the force balance in the numerical model of TCS due to the smooth and accurate approximation of the second moments of the proton distribution function is fulfilled with very high accuracy. This suggests the configurations obtained are stationary and the numerical model is of high quality. Note that in Figure 1 the current density and concentration profiles in the center of the sheet have a characteristic bifurcation as the forked tongue of a snake. As shown in [Zelenyi et al., 2004; Zelenyi et al., 2011; Sitnov et al., 2000], this bifurcation is caused by the presence of a relatively large fraction of quasi-trapped protons, which oscillate in the center of the sheet long enough. This bifurcation is enhanced as the fraction of such protons increases. The reflection of this subtle effect in the simulation results also confirms the high quality of the numerical model.

Note also that in many numerical models using the particle-in-cell method, the second moments of distribution
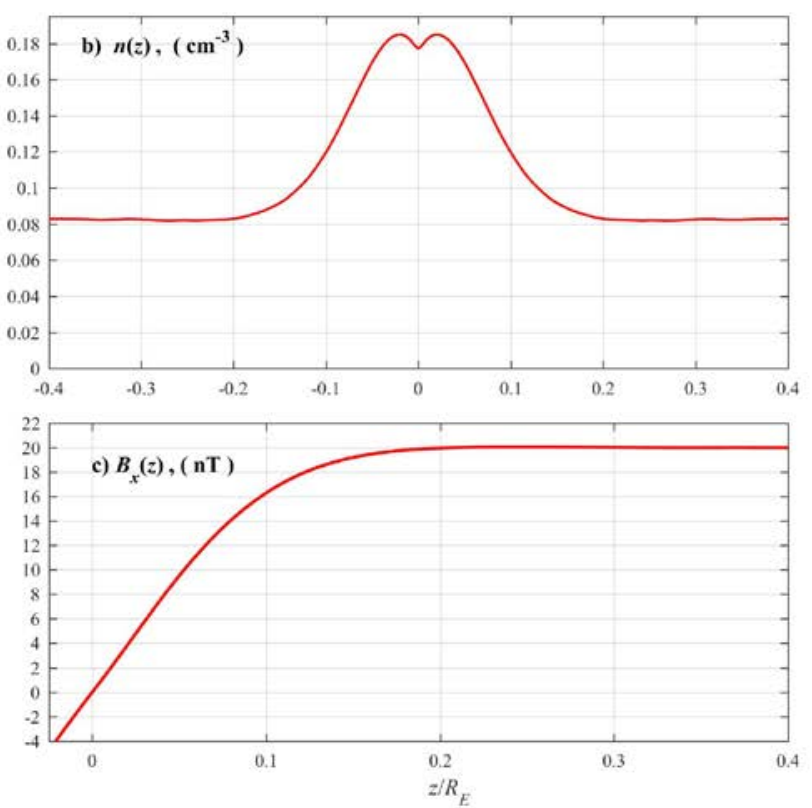

Figure 1. Symmetric configuration: the proton current density $y$ component $j_{\text {iy }}(z)(a)$; the proton density $n(z)(b)$; the magnetic field component $B_{x}(z)(c)$ (in view of the symmetry, its right side is shown for $z / R_{\mathrm{E}} \geq-0.025$ ). $\delta=V_{D} / V_{\mathrm{Tp} 0}=2.5$ 

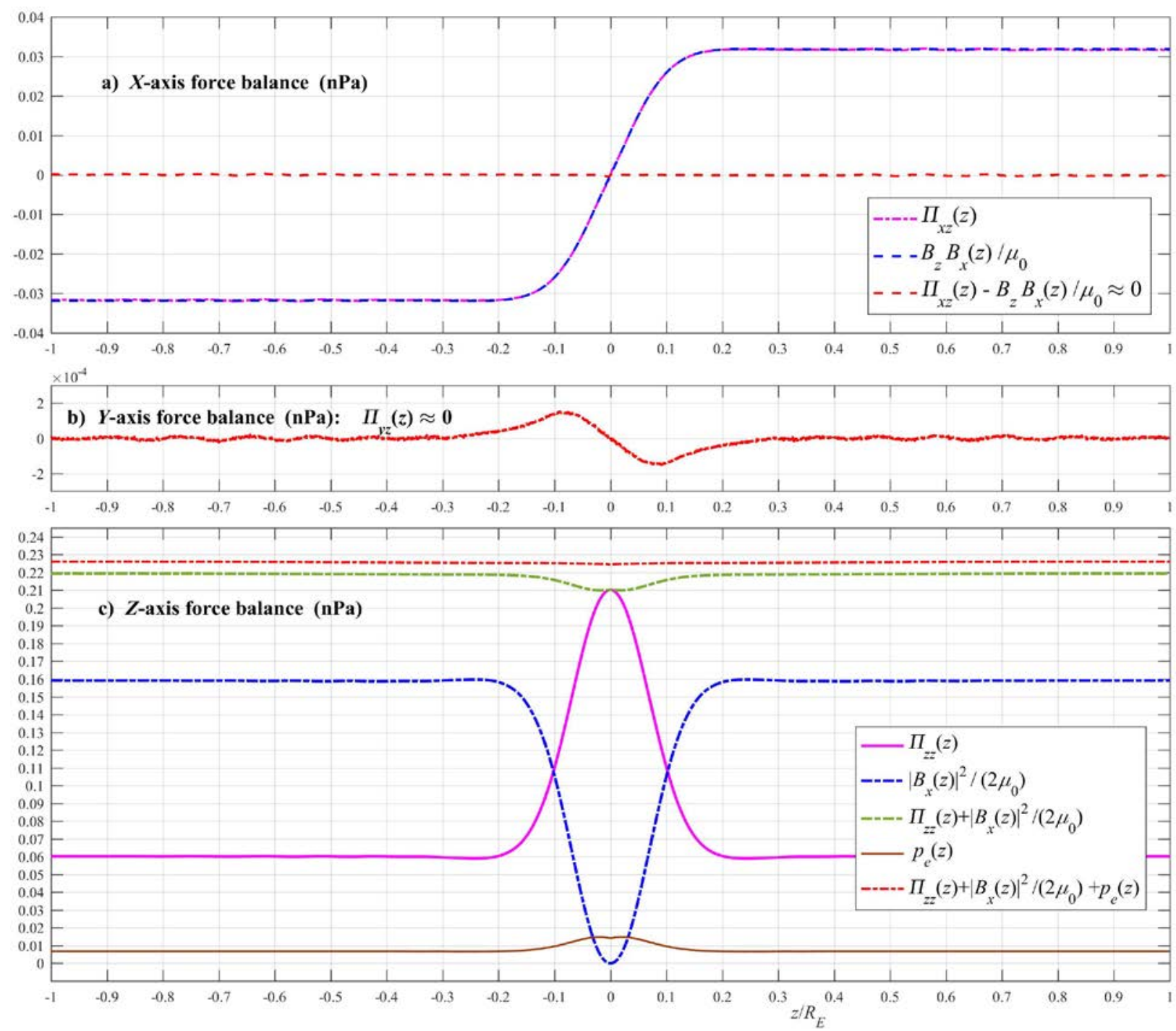

Figure 2. Force balance (in nanopaskal). The total balance is indicated by the red line

The electric field potential is also roughly constant, i.e. the electric field is very small and has almost no effect on the TCS configuration compared to the aforementioned symmetric configurations. Figure 3 presents the stationary configuration in which $\delta=V_{D} / V_{\mathrm{Tp} 0}=2$, the shear magnetic field component is equal to zero outside the sheet, and all other input parameters have the values defined at the beginning of this section. Comparison between Figures 1 and 3 shows that in the configuration obtained for the equal total current across CS, i.e. for the same change in the tangential magnetic field component when passing through CS $\Delta B_{x}=B_{x}(L)-B_{x}(-L)=2 B_{x 0}$, the current sheet became approximately three times wider, and the maximum current density component $j_{y}(z)$ decreased approximately threefold.

\section{CONCLUSION}

In this paper, we have reported three new results. The first result concerns divergent forms of force balance equations in collisionless plasma: Formulas (1.12) and (1.13) are for the general case; and Formulas (1.15) and
(1.17), for magnetized electrons. In the case of plasma consisting of protons and magnetized electrons, Equations (1.15) and (1.17) have the form of (1.18) and (1.19) respectively and are divergent forms of the proton momentum flux equation (motion equation). The solar wind plasma and Earth's magnetosphere plasma, under quiet heliogeophysical conditions mainly involve protons and magnetized electrons. Equations (1.18) and (1.19) may therefore be useful for determining the position and shape of the bow shock and the magnetopause of Earth's magnetosphere. In addition, Equations (1.18) and (1.19) can be used to analyze measurement data from the MMS spacecraft mission. They may also be utilized to delve into the features of large-scale inhomogeneous magnetoplasma structures in the solar wind, such as current sheets and magnetic islands.

The second result deals with force balance equation (2.3) in the form of a conservation law for stationary spatially one-dimensional TCS.

This equation is necessary to impose boundary conditions in an asymmetric case, as well as to check the stationarity of the resulting numerical solution.

Examples of such a check are given in Figures 2 and 4 . 

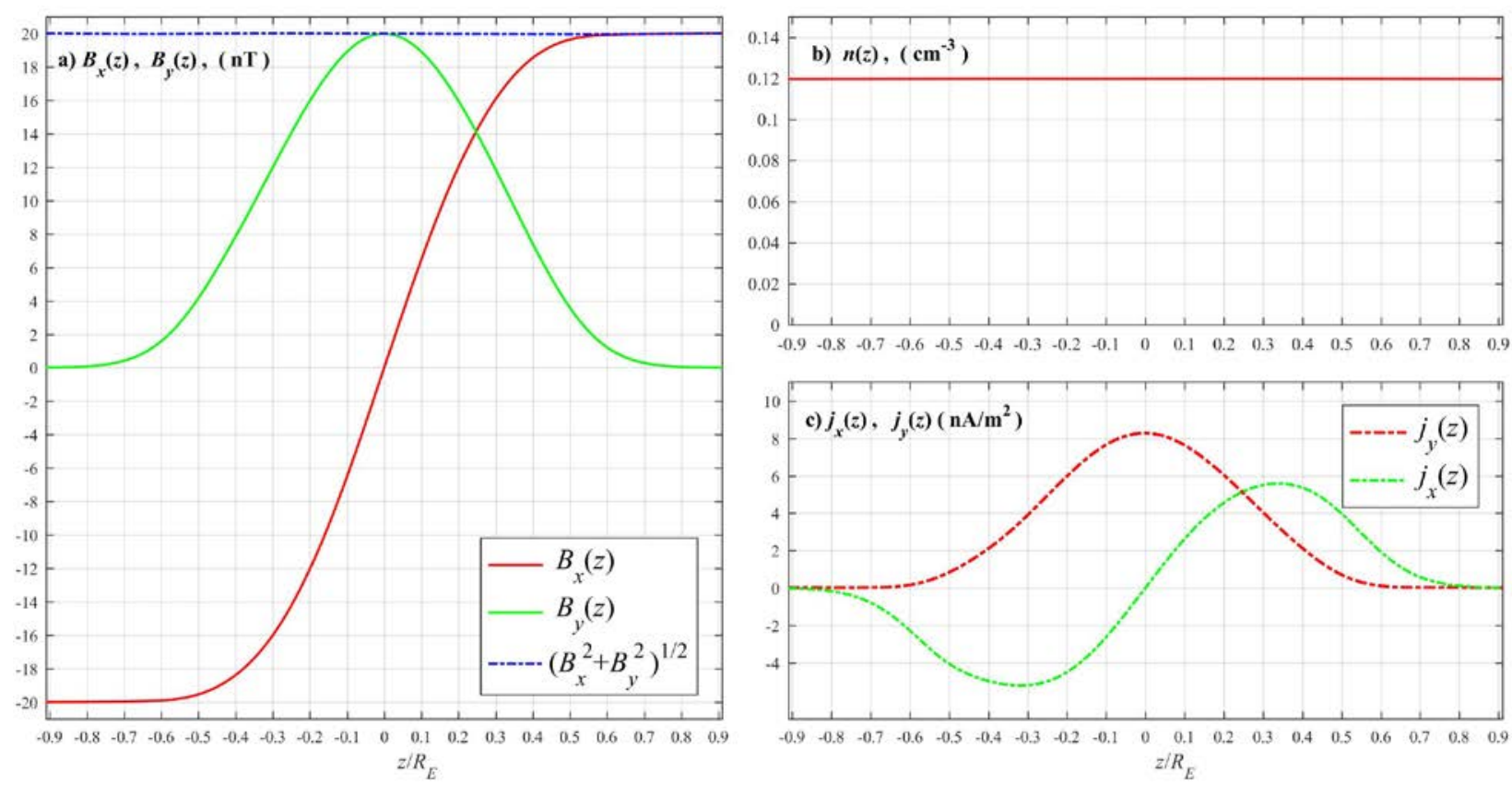

Figure 3. Profiles of magnetic field components $(a)$; density $n(z)(b)$, and current density components $j_{x}(z)$ and $j_{y}(z)(c)$
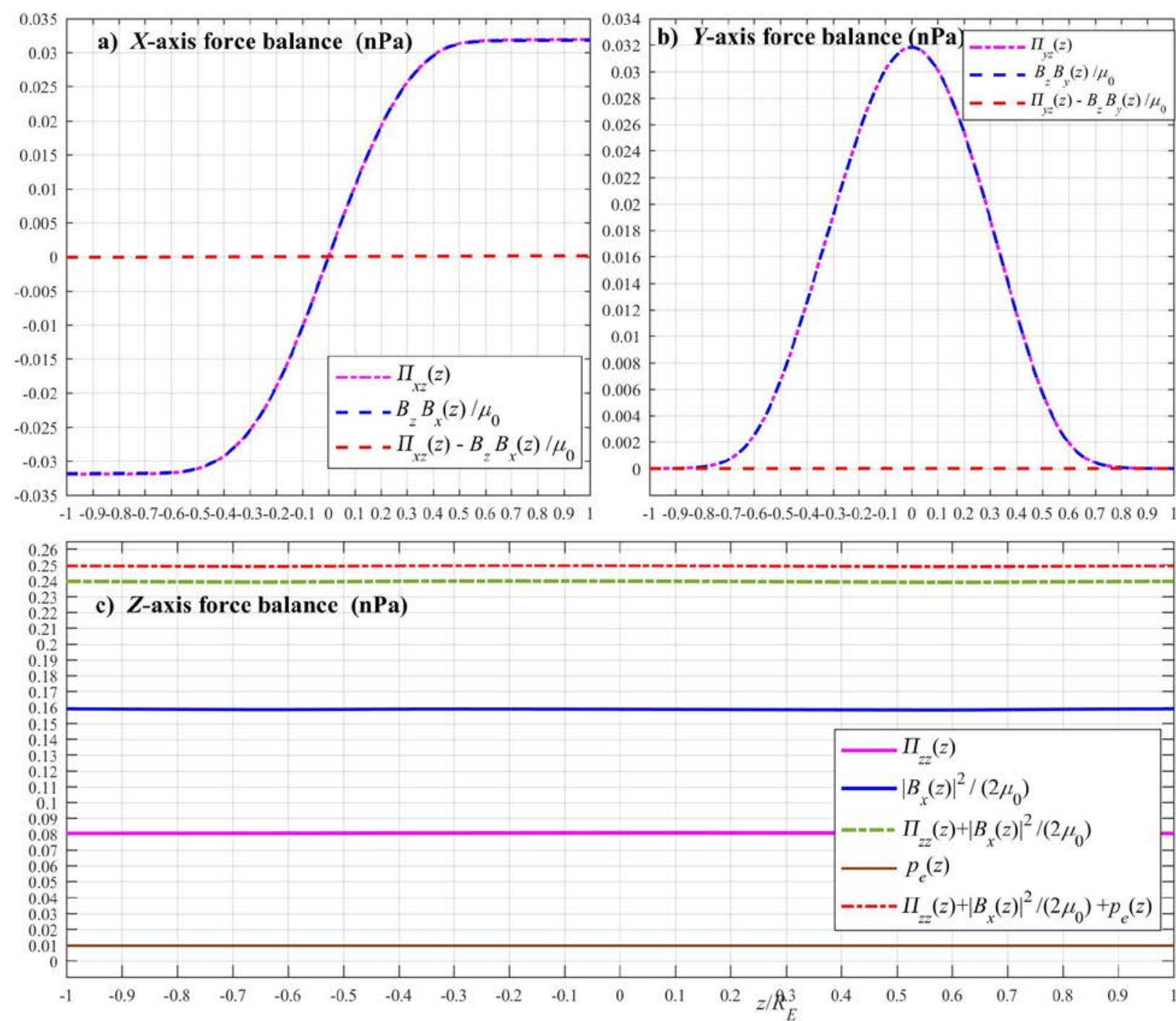

Figure 4. Force balance for $\delta=V_{D} / V_{\mathrm{Tp} 0}=2$ 
Note that this equation allows us to consider CS with ions of several types. An interesting example is CS in Jupiter's magnetotail (see [Artemyev et al., 2014] and references therein), wherein along with protons there are hot oxygen ions $\mathrm{O}^{+}$and hot sulfur ions $\mathrm{S}^{+}$, which can significantly affect the structure of the CS. Another example is CS of near and middle Earth's magnetotail during magnetic disturbances with injection of oxygen ions $\mathrm{O}^{+}$from the ionosphere into the magnetosphere. In this case, unlike quiet conditions, the oxygen ions $\mathrm{O}^{+}$, which can significantly alter the configuration of this CS, prevail in it; The final result fully describes the current of magnetized electrons in the numerical model of TCS with the given normal magnetic field component - (3.9), (3.21), (3.22). Mingalev et al. [2018] have published only expression (3.22) for the electron current $y$-component in a less convenient form. In the most advanced analytical models of TCS, which are described in the reviews [Zelenyi et al., 2011, 2016], the electron current is calculated assuming that both temperatures of magnetized electrons are constant: $T_{\mathrm{e} \|} \equiv$ const,$T_{\mathrm{e} \perp} \equiv$ const. In [Mingalev et al., 2018] and in this paper, this assumption is refined based on the kinetic description of magnetized electrons, which confirms that the longitudinal temperature is constant, but the orthogonal temperature varies according to Formula (3.19).

\section{REFERENCES}

Arons J. A tale of two current sheets. High-energy emission from pulsars and their systems. Part of the Astrophysics and Space Science Proceedings book series (ASSSP). Springer Verlag Berlin Heidelberg, 2011, p. 165.

Artemyev A.V. A model of one-dimensional current sheet with parallel currents and normal component of magnetic field. Physics of Plasmas. 2011, vol. 18, p. 022104. DOI: 10.1063/ 1.3552141.

Artemyev A.V., Zelenyi L.M., Kinetic structure of current sheets in the Earth magnetotail. Space Sci. Rev. 2013, vol. 178, iss. 2-4, pp. 419-440. DOI: 10.1007/s11214-012-9954-5.

Artemyev A.V., Petrukovich A.A., Frank A.G., Nakamura R., Zelenyi L.M. Intense current sheets in the magnetotail: Peculiarities of electron physics. J. Geophys. Res. 2013, vol. 118, pp. 2789-2799. DOI: 10.1002/jgra.50297.

Artemyev A.V., Vasko I.Yu., Kasahara S. Thin current sheets in the Jovian magnetotail. Planetary and Space Sci. 2014, vol. 96, pp. 133-145. DOI: 10.1016/j.pss.2014.03.012.

Ashour-Abdalla M., Zelenyi L.M., Peroomian V., Richard R.L. Consequences of magnetotail ion dynamics. J. Geophys. Res. 1994, vol. 99, no. A8, pp. 14891-14916. DOI: 10.1029/94JA00141.

Baumjohann W. Roux A., Le Contel O. et al., Dynamics of thin current sheets: Cluster observations. Ann. Geophys. 2007, vol. 25, iss. 6, pp. 1365-1389. DOI: 10.5194/angeo-251365-2007.

Bykov A.A., Zelenyi L.M., Malova Kh.V. Triple splitting of a thin current sheet: A new type of plasma equilibrium. Plasma Phys. Rep. 2008, vol. 34, no. 2, pp. 128-134. DOI: 10.1007/s11452-008-2005-5.

Bykov A.A., Ermakova K.E. A self-consistent model for the electronic component of a thin current sheet in the Earth's magnetosphere. Moscow University Physics Bull. 2016, vol. 71, pp. 43-51.
Frank A.G., Artemyev A.V., Zelenyi L.M. Current sheets in the Earth's magnetosphere and in laboratory experiments: The magnetic field structure and the Hall effect. J. Exp. Theor. Phys. 2016, vol. 123, pp. 699-715. DOI: 10.1134/S1063776116090119.

Grigorenko E.E., Malova H.V., Malykhin A.Y., Zelenyi L.M. A possible mechanism of the enhancement and maintenance of the shear magnetic field component in the current sheet of the Earth's magnetotail. Plasma Phys. Rep. 2015, vol. 41, pp. 88-101. DOI: 10.1134/S1063780X1501002X.

Harris E.G. On a Plasma sheath separating regions of oppositely directed magnetic fields. Il Nuovo Cimento. 1962, vol. 23, iss. 1, pp. P. 115-119.

Ilgisonis V.I. Guiding-center theory for three-dimensional collisionless finite Larmor radius plasmas. Phys. Fluids B.: Plasma Phys. 1993, vol. 5, iss. 7, p. 2387. DOI: 10.1063/ 1.860722 .

Kalsrud R. Fundamentals of Plasma Physics. Ed. A.A. Galeev, R. Sudan. Moscow: Energoatomizdat, 1983. vol. 1, pp. 122-152. (In Russian).

Kocharovsky V.V., Martyanov V Yu, Tarasov S.V. Analytical theory of self-consistent current structures in a collisionless plasma. Physics-Uspekhi, 2016, vol. 59, no. 12, pp. 1165-1210. DOI: 10.1134/ S1063773719080048.

Kocharovsky V.V., Kocharovsky Vl.V., Martyanov V.Y., Nechaev A.A. An analytical model for the current structure of the magnetosheath boundary in a collisionless plasma. Astronomy Lett. 2019, vol. 45, iss. 8, pp. 551-564. DOI: 10.1134/ S1063773719080048.

Kropotkin A.P., Lui A.T.Y. Quasi-static evolution of the magnetosphere: The substorm growth phase. J. Geophys. Res. 1995, vol. 100, iss. A9, pp. 17231-17240. DOI: 10.1029/ 95JA00792.

Malova H.V., Mingalev O.V., Grigorenko E.E., Mingalev I.V., Melnik M.N., Popov V.Y., et. al. Formation of selforganized shear structures in thin current sheets. J. Geophys. Res.: Space Phys. 2015, vol. 120, pp. 4802-4824. DOI: 10.1002/ 2014JA020974.

Malova H.V., Popov V.Yu., Grigorenko E.E., Petrukovich A.A., Delcourt D., Sharma A.S., et al. Evidence for quasiadiabatic motion of charged particles in strong current sheets in the solar wind. Astrophys. J. 2017, vol. 834, no. 1, p. 34. DOI: 10.3847/1538-4357/834/1/34.

Mingalev O.V., Mingalev I.V., Mel'nik M.N., Artemyev A.V., Malova H.V., Popov V.Yu., et al. Kinetic models of current sheets with a sheared magnetic field. Plasma Phys. Rep. 2012, vol. 38, pp. 300-314. DOI: 10.1134/S1063780X12030063.

Mingalev O.V., Malova H.V., Mingalev I.V., Mel'nik M.N., Setsko P.V., Zelenyi L.M. Model of a thin current sheet in the Earth's Magnetotail with a kinetic description of magnetized electrons. Plasma Phys. Rep. 2018, vol. 44, pp. 899919. DOI: $10.1134 / \mathrm{S} 1063780 X 18100082$.

Mingalev O.V., Mingalev I.V., Malova H.V., Merzlyi A.M., Mingalev V.S., Khabarova O.V. Description of large-scale processes in the near-Earth space plasma. Plasma Phys. Rep. 2020, vol. 46, pp. 374-395. DOI: 10.1134/S1063780X20030083.

McPherron R.L., Nishida A., Russell C.T. Is near-Earth current sheet thinning the cause of auroral substorm onset? Quantitative Modeling of Magnetosphere-Ionosphere Coupling Processes. Ed. by Y. Kamide, R.A. Wolf. Kyoto Sangyo University: Kyoto, Japan, 1987, pp. 252-265.

Morozov A.I., Solovyov L.S.Questions of the Theory of Plasma. Ed. M.A. Leontovich. Moscow, Gosatomizdat, 1963, iss. 2, pp. 177-255. (In Russian).

Rudakov L.I. Sagdeev R.Z. Plasma Physics and the Problem of Controlled Thermonuclear Reactions. Ed. M.A. Leon- 
tovich. Moscow, Academy of Sciences of the USSR Publ., 1958, vol 3, 268 p. (In Russian).

Runov A., Sergeev V.A., Nakamura R., Baumjohann W., Apatenkov S., Asano Y., et al. Local structure of the magnetotail current sheet: 2001 Cluster observations. Ann. Geophys. 2006, vol. 24, iss. 1, pp. 247-262. DOI: 10.5194/angeo-24247-2006.

Sergeev V.A., Mitchell D.G., Russell C.T., Williams D.J. Structure of the tail plasma/current sheet at $11 \mathrm{R}_{\mathrm{E}}$ and its changes in the course of a substorm. J. Geophys. Res. 1993, vol. 98, pp. 17345-17365. DOI: 10.1029/93JA01151.

Sergeev V.A., Pulkkinen T.I., Pellinen R.J. Coupled-mode scenario for the magnetospheric dynamics. J. Geophys. Res. 1996, vol. 101, iss. A6, pp. 13047-13066. DOI: 10.1029/ 95JA03192.

Sitnov M.I., Zelenyi L.M., Malova H.V. Sharma A.S. Thin current sheet embedded within a thicker plasma sheet: selfconsistent kinetic theory. J. Geophys. Res. 2000, vol. 105, no. A6, pp. 13029-13044. DOI: 10.1029/1999JA000431.

Somov B.V. Verneta A.I. Tearing instability of reconnecting current sheets in space plasmas. Space Sci. Rev. 1993, vol. 65, pp. 253-288. DOI: 10.1007/BF00754510.

Speiser T.W., Particle trajectories in model current sheets; 1. Analytical solutions. J. Geophys. Res. 1965, vol. 70, pp. 4219-4226.

Volkov T.F. Hydrodynamic description of a highly rarefied plasma. Questions of the Theory of Plasma. Ed. M.A.
Leontovich. M: Gosatomizdat, 1964, vol. 4, pp. 3-19. (In Russian).

Zelenyi L.M., Malova H.V., Popov V.Yu. Delcourt D., Sharma A.S. Nonlinear equilibrium structure of thin currents sheets: influence of electron pressure anisotropy. Nonlinear Processes in Geophysics, European Geosciences Union (EGU), 2004, vol. 11, no. 5/6, pp. 579-587.

Zelenyi, L.M., Malova, H.V., Artemyev, A.V., Popov V.Yu., Petrukovich A.A. Thin current sheets in collisionless plasma: Equilibrium structure, plasma instabilities, and particle acceleration. Plasma Phys. Rep. 2011, vol. 37, pp. 118-160. DOI: 10.1134/S1063780X1102005X.

Zelenyi L.M., Malova H.V., Grigorenko E.E., Popov V.Yu. Thin current sheets: from Ginzburg - Syrovatsky up to the present days. Physics-Uspekhi. 2016, vol. 59, iss. 11, pp. 1057-1090. DOI: 10.3367/UFNe.2016.09.037923.

How to cite this article

Mingalev O.V., Setsko P.V., Melnik M.N., Mingalev I.V., Malova H.V., Merzlyi A.M. Force balance in current sheets in collisionless plasma. Solar-Terrestrial Physics. 2021. Vol. 7. Iss. 2. P. 11-21. DOI: $10.12737 /$ stp-72202102. 